\title{
The Aphid Fauna (Hemiptera:Aphidoidea) and Host Plants of The Büyükada Island (İstanbul, Turkey)
}

\author{
Hayal Akyıldırım Beğen ${ }^{1 *}$, Gazi Görür ${ }^{2}$ \\ ${ }^{1}$ Vocational School of Health Services, Artvin Çoruh University, Artvin, Turkey \\ ${ }^{2}$ Department of Biotechnology, Faculty of Arts and Sciences, Niğde Ömer Halis Demir University, Niğde, Turkey
}

\begin{abstract}
Article History
Received:

19.05.2020

Accepted: $\quad 23.12 .2020$

Published: $\quad 20.03 .2021$

Research Article

Abstract - About 5100 aphid (Hemiptera: Aphidoidea) species that feed on different host plants have spread all over the world. About 5000 aphid taxon feed on different plant species. Aphids are small, soft-bodied and one of the sap-feeding insect groups and also can damage almost all plants. Some aphid species are specific to only one host plant, while some feed on numerous host plants. In this study, aphid fauna and host plants of aphids were determined in Büyükada islands of İstanbul was determined in this study. Aphid species were distributed from cultivated plants, wild trees and shrubs in the area. During these study, 150 aphid samples were collected in the area. Apterae and alatae individuals of aphid samples were collected as much as possible from aphid colonies on cultivated and ornamental plants. 43 aphid taxa including two new records Turkey aphid fauna ( 1 family, 6 subfamilya, 6 tribus ve 23 genus) were determined on 46 different plant species at the end of the study. Malva neglecta, Nerium oleander, and Pinus spp. are the most sampled plant species. Aphis craccivora, Aphis fabae, and A. spiracola are the most determined aphid species in the area. All defined aphid species are the first records for the Büyükada island. Findings of the presented study and other recent studies showed that with the detailed studies, Turkey aphid fauna will be substantially increased thanks to the more detailed local studies.
\end{abstract}

Keywords - Aphid, Büyükada, İstanbul, plant, Turkey

\section{Introduction}

Aphids are considered remarkable pest that feeds on plant sap and damages them directly by sucking their phloem sap, indirectly by transmitting pathogens around the world. About 5100 aphid species have been identified around the world, and 1600 aphid species are present in Europe (Favret, 2020; Blackman and Eastop, 2020). Preliminary studies related to Turkey aphid fauna date back to twentieth-century (Fahringer, 1922).

Çanakçığlu (1975) summarised all studies on aphids and published nearly about 260 aphid species.

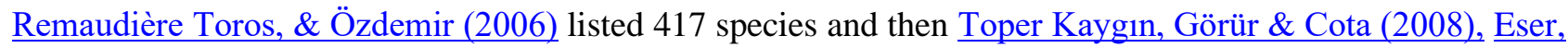
Görür, Tepecik \& Akyıldırım (2008), Görür, Zeybekoğlu, Akyürek, Işık \& Akyıldırım (2009), Akyürek, Zeybekoğlu \& Görür $(2010,2011)$, Görür, Akyıldırım Beğen, Olcabey \& Akyurek (2012) published 480 aphid taxa including 2 new records from our study area which were Acyrthosiphon kondoi Shinji, 1938 and Eulachnus pumilae Inouye, 1939. Barjadze, Özdemir \& Blackman. (2014) published Aphis matricariae Barjadze \& Özdemir, 2014 and Protaphis kvavadzei Barjadze \& Özdemir, 2014 as new records. Moreover,

\footnotetext{
1 (iD) h.akyildirim@artvin.edu.tr

2 (iD) ggorur@ohu.edu.tr

*Corresponding Author
} 
Barjadze, Blackman \& Özdemir (2015) was defined Aphis polii on Teucrium sp. from Turkey. Görür, Toper Kaygin, Senol \& Akyildirim Beğen (2015) shown that Cinara curvipes (Patch, 1912) was invasive and a new addition for Turkey. Șenol, Akyıldırım Beğen, Görür \& Demirtaș (2015a,b) described new records (26 species) and increased the number of the aphid fauna to 532. Kök, Kasap \& Özdemir (2016) and Kök \& Kasap (2019) added 2 aphid species and the number reached to 534. Görür, Şenol \& Aky1ldırım Beğen (2019) listed 40 new aphid records to the aphidofauna. Recent reanalysis of the aphid species and determined host plants clearly showed that the number of the aphid taxa in Turkey reached more than 570 (Görür Șenol \& Akyıldırım Beğen, 2019; Görür, Șenol, Akyıldırım Beğen \& Akyürek, 2020).

\section{Materials and Methods}

\subsection{Field Introduction}

The Princes Islands include nine islands, four of them are large (Büyükada, Heybeliada, Burgazada, and Kınalıada) and 5 of them are smaller (Yassıada, Sivriada, Kaşık Adası, Sedef Adası and Tavşan Adası), placed in the southeast part of the Marmara Sea of Istanbul. Büyükada, the area of this study is the biggest island among them (Figure 1).

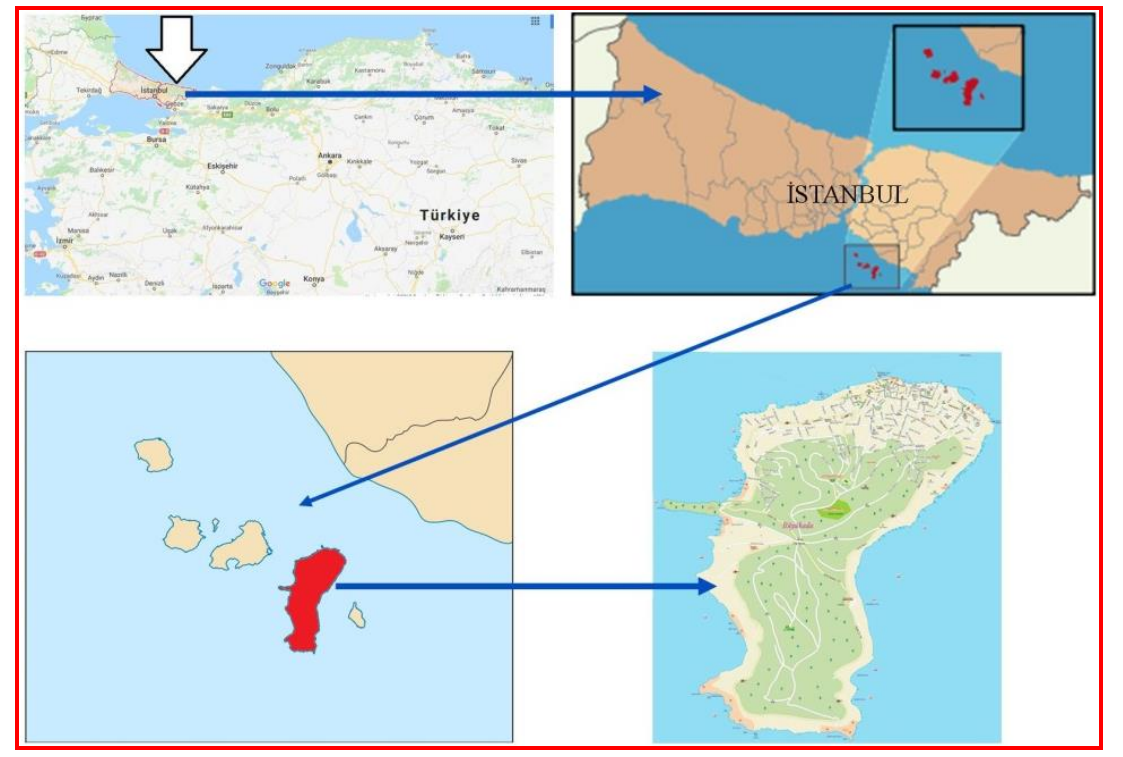

Figure 1. Location of Büyükada Island

The annual average temperature of these Islands is $14.4^{\circ} \mathrm{C}$, generally above $25^{\circ} \mathrm{C}$ during May-September. The annual average precipitation is about $823.0 \mathrm{~mm}$. The relative humidity is between $73-77 \%$ that decreases to around 65-68\% during summer (MGM, 2020). Büyükada island has natural and exotic plant species due to location and climatic types of the area. Blacksea and Mediterranian climatic types are observed in the area (Kaya \& Aladağ, 2009). Exotic plants grown throughout the island, especially in the gardens, parks, and even roadsides in residential areas.

\subsection{Methods}

The field studies were conducted in Büyükada Islands from April to October 2010 (Figure 1). Focused on the sample possible higher amount of all forms (apterae and alatae individuals) on host plants. Specimens were preserved in an eppendorf tube containing 96\% alcohol. Samples were picked up from almost all naturally grown and agricultural plants. Collection and preparation of the aphid samples have been carried out in accordance with the methods of Martin (1983). Species were described based on Blackman \& Eastop (2020) and Çanakçığlu (1975). Systematic status and host plants of the identified aphids were determined 
from Blackman and Eastop (2020) and Holman (2009). When defining all aphid samples, all books and papers published about diagnostic characters, determined plant species, origination and recent dispersal area were examined carefully. Definition, taxonomic status and distribution informations controlled by related literature (Holman, 2009; Favret, 2020; Blackman \& Eastop, 2020). All collected aphid specimens were kept in the Aphid laboratory of Faculty of Arts and Sciences, Niğde Ömer Halis Demir University.

\section{Results and Discussion}

150 aphid population were sampled and 43 aphid species ( 23 genera, 6 tribe) belonging to 6 subfamilies were determined from the study area (Figure 1). Identified taxa were edited alphabetically and listed (Table 1). Photos of some important species were given (Figure 2,3 ).

Among these identified species, two new records were determined for Turkey and added to a recent checklist of the Turkey aphid fauna (Görür et al., 2012). The first of these is Acyrthosiphon kondoi Shinji, 1938 and collected on Malva sp. (Malvaceae) second one is Eulachnus pumilae Inouye, 1939 and collected on Pinus brutia (Pinaceae). All taxonomical name lists, host plants and dates of the determined species from study area were given. Determined species are listed alphabetically.

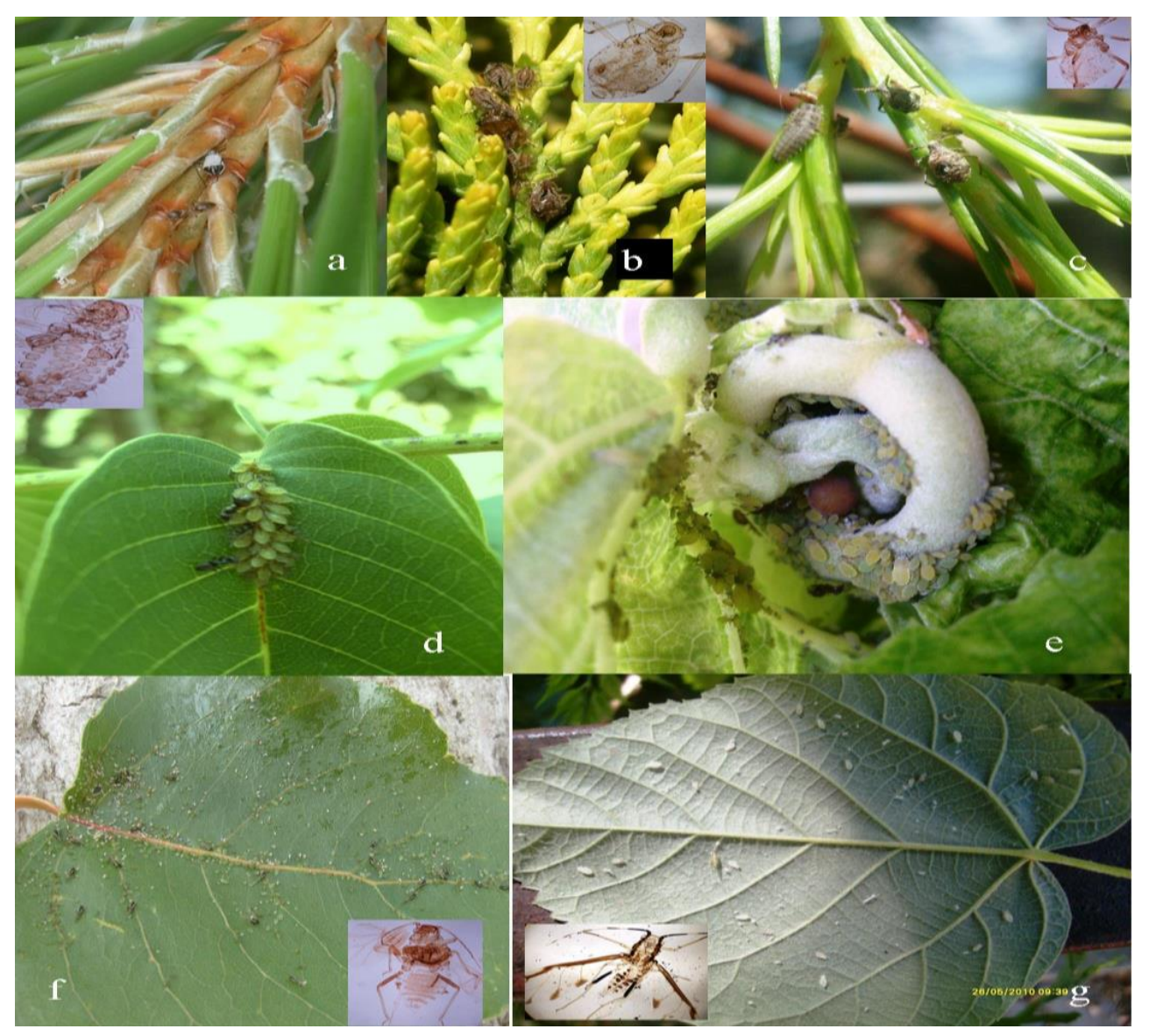

Figure 2. Aphid species on host plants (a- Cinara brauni on Pinus sp.; b- Cinara tujafilina on Platycladus orientalis;c- Cinara maghrebica on Pinus sp. ;d- Panaphis juglandis on Juglans regia ;e- Patchiella reaumuri on Tilia sp. ;f- Chaitophorus populialbae on Populus nigra; g- Eucallipterus tiliae on Tilia sp.) 
Acyrthosiphon euphorbiae Börner, 1940.

Examined specimen: Collected on

Euphorbia sp.on 11.05.2010.

\section{A. kondoi Shinji, 1938}

Examined specimen: Collected on Lathyrus sp.on 21.05.2010

A. malvae (Mosley, 1841)

Examined specimen: Collected on Senecio pseudo-orientalis on 11.05.2010; Malva sp. on 14.05.2010.

Aphis craccivora Koch 1854

Examined specimen: Collected on Citrus sp. (Family) on 11.V.2010, Acacia sp. on 22.06.2010; Portulacca oleracea, Wistera sp. on 28.06.2010-02.07.2010.

\section{A. fabae Scopoli 1763}

Examined specimen: Sampled on Hibiscus syriacus (ağaç hatmi); Hedera helix on 10.05.2010 Polygonum sp.(çobandeğneği); Urtica sp.(1sirgan otu); Anthemis sp. on 11.05.2010;

Nerium oleander on 12.05.2010; Hedera sp.

Polygonum sp.on 14.05.2010;

A. gossypii Glover 1877

Examined specimen: Collected on Punica granatum on 26.05.2010.

\section{A. hederae Kaltenbach, 1843}

Examined specimen: Collected on Hedera helix 07.07.2010.

A. molluginis (Börner, 1950)

Examined specimen: Collected on Galium aparine on 10-11.05.2010.

\section{A. nasturtii Kaltenbach, 1843}

Examined specimen: Collected on Rumex sp. on 28.06.2010.

\section{A. nerii Boyer de Fonscolombe, 1841}

Examined specimen: Found on Nerium oleander on 10.05.2010; 11.05.2010; 27.06.2010

\section{A. pomi de Geer, 1773}

Examined specimen: Collected on Pyrus sp. on 26.05.2010

A. ruborum (Börner, 1932)

Examined specimen: Found on Rubus sp. on 27.06.2010.

\section{A. spiraecola Patch, 1914}

Examined specimen: Collected on

Cotoneaster franchetti on 10.05.2010;
Chenopodium sp. on 11.05.2010; 12.05.2010; Nerium oleander on 21.05.2010; Trifolium sp. on 22.06.2010; 28.06.2010.

\section{A. umbrella (Börner, 1950)}

Examined specimen: Collected on Malva sp.on 07.07.2010.

Aulocorthum solani (Kaltenbach, 1843)

Examined specimen: Collected on Ulmus sp. on 10.05.2010.

Brachycaudus cardui (Linnaeus, 1758)

Examined specimen: Collected on Carduus sp.on 14.05.2010; Carthamus sp. on 21.05.2010;

Carduus acanthoides on 26.05.2010.

B. helichrysi (Kaltenbach, 1843)

Examined specimen: Collected on

Cynoglossum sp.on 12.05.2010 ;

14.05.2010; 21.05.2010;

Lycopersicum esculentum on 02.07.2010.

Brevicoryne brassicae (Linnaeus, 1758)

Examined specimen: Collected on Bunias orientalis on 17.05.2010.

Chaitophorus populialbae (Boyer de

Fonscolombe, 1841)

Examined specimen: Collected on Populus nigra on 21.05.2010.

Cinara tujafilina del Guercio, 1909

Examined specimen:: Collected on

Platycladus orientalis on 10.05.2010;

21.05.2010.

C. brauni Börner, 1940

Examined specimen: Collected on Pinus sp. on 28.06.2010.

C. maghrebica Mimeur, 1934

Examined specimen: Collected on Pinus sp. on 26.05.2010;27.06.2010.

C. pilicornis (Hartig, 1841)

Examined specimen: Collected on Picea sp. on 27.06.2010.

C. pruinosa (Hartig, 1841)

Examined specimen: Collected on Picea sp. on 28.06.2010.

Drepanosiphum oregonensis Granovsky, 1939

Examined specimen:: Collected on Acer

trautvetteri on 26.05.2010

Eucallipterus tiliae (Linnaeaus, 1758) 
Examined specimen: Collected on Tilia sp. on 12.05.2010; 26.05.2010 28.06.2010.

*Eulachnus pumilae Inouye, 1939

Examined specimen: Collected on Pinus sp. on 27.06.2010.

Hyalopterus pruni (Geoffroy, 1762)

Examined specimen: Collected on Prunus domestica on 12.05.2010.

Liosomaphis berberidis (Kaltenbach, 1843) Examined specimen: Collected on Berberis thunbergii on 12.05.2010.

Lipaphis erysimi (Kaltenbach, 1843)

Examined specimen: Collected on

Brassica nigra on 21.05.2010.

Macrosiphum euphorbia (Thomas, 1878)

Examined specimen: Collected on Urtica

sp. on 10.05.2010.

M. impatientis Williams, 1911.

Examined specimen: Collected on Rosa

canina on 14.05.2010.

M. rosae (Linnaeus, 1758)

Examined specimen: Collected on Rosa sp. on $11.05 .2010 ; 12.05 .2010 ; 02.07 .2010$

;07.07.2010

Myzus persicae (Sulzer, 1776)

Examined specimen: Collected on Malva sp. on 21.V.2010.

Ovatomyzus chamaedrysi (Passerini,1879)

Examined specimen: Collected on Mentha sp. on 14.05.2010.

Panaphis juglandis (Goeze, 1778)

Examined specimen: Collected on Juglans regia on 07.07.2010.

Patchiella reaumuri (Kaltenbach, 1843)

Examined specimen: Collected on Tilia sp. on 10.05.2010.

Rhopalosiphum padi (Linnaeus, 1758)

Examined specimen: Collected on

Hordeum murinumon 02.07.2010.

Schizaphis rotundiventris (Signoret, 1860)

Examined specimen: Sampled on Palm sp.on 22.06.2010; 27.06.2010.

Sitobion avenae (Fabricius, 1775)

Examined specimen: Collected on

Cynodon

dactylon on 07.07.2010.

S. fragariae (Walker, 1848)
Examined specimen: Collected on

Hordeum sp. on 14.05.2010; Poa sp. on

21.05.2010; Bromus sp. on 26.05.2010.

Uroleucon sonchi (Linnaeus, 1767)

Examined specimen: Collected on

Chondrilla junce on 10.05.2010.

Wahlgreniella nervata (Gillette, 1908)

Examined specimen: Collected on Sonchus sp. on 07.07.2010. 


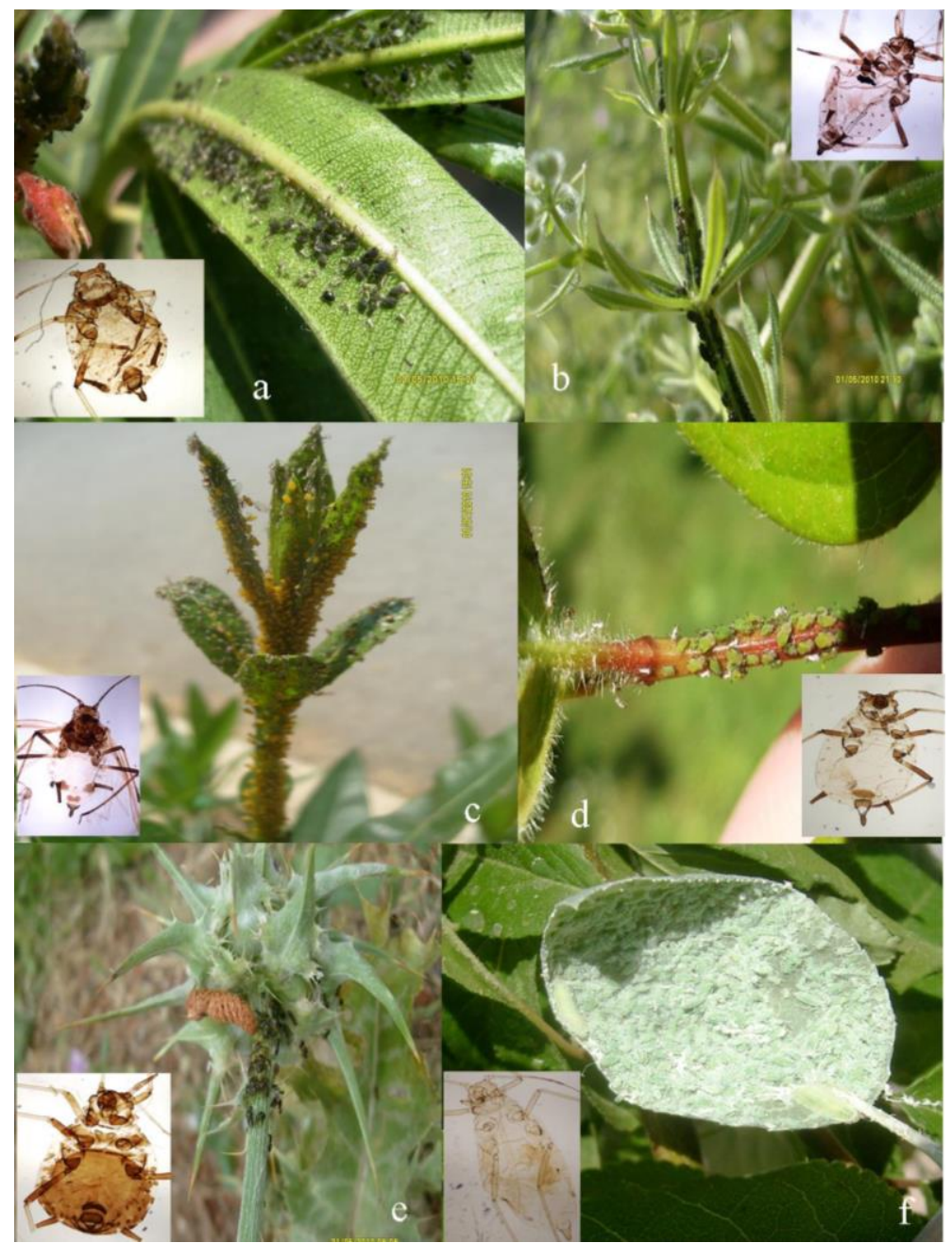

Figure 3. Determined species on host (a- Aphis fabae on Nerium oleander;b- A. molluginis on Gallium aparine;c- A. nerii on Nerium oleander;d- Aphis spiraecola on Cotoneaster sp.;e- Brachycaudus cardui on Carduus sp.;f- Hyalopterus pruni on Prunus domestica) 
Table 1

Determined aphid species and host plants

\begin{tabular}{|c|c|c|c|}
\hline APHID SPECIES & HOST PLANTS & APHID SPECIES & $\begin{array}{l}\text { HOST } \\
\text { PLANTS }\end{array}$ \\
\hline $\begin{array}{l}\text { Acyrthosiphon euphorbiae } \\
\text { Börner, } 1949\end{array}$ & Euphorbia sp. & $\begin{array}{l}\text { C. pilicornis (Hartig, } \\
\text { 1841) }\end{array}$ & Picea sp. \\
\hline A. kondoi Shinji, 1938* & Lathyrus sp. & $\begin{array}{l}\text { C. pruinosa (Hartig, } \\
\text { 1841) }\end{array}$ & Picea sp. \\
\hline A. malvae (Mosley, 1841) & $\begin{array}{l}\text { Malva neglecta. } \\
\text { Senecio pseudo- } \\
\text { orientalis }\end{array}$ & $\begin{array}{l}\text { Drepanosiphum } \\
\text { oregonensis } \\
\text { Granovsky, } 1939\end{array}$ & Acer trautvetteri \\
\hline $\begin{array}{l}\text { Aphis craccivora Koch, } \\
1854\end{array}$ & $\begin{array}{l}\text { Robinia } \\
\text { pseudoacacia } \\
\text { Wistera floribunda } \\
\text { Lycopersicum } \\
\text { esculentum } \\
\text { Portulaca oleracea }\end{array}$ & $\begin{array}{l}\text { Eucallipterus tiliae } \\
\text { (Linnaeaus, 1758) }\end{array}$ & Tilia sp. \\
\hline A. fabae Scopoli, 1763 & $\begin{array}{l}\text { Hibiscus syriacus } \\
\text { Hedera helix } \\
\text { Polygonum } \mathrm{sp} . \\
\text { Urtica dioica } \\
\text { Anthemis } \mathrm{sp} . \\
\text { Nerium oleander }\end{array}$ & $\begin{array}{l}\text { Eulachnus pumilae } \\
\text { Inouye, 1939* }\end{array}$ & Pinus sp. \\
\hline A. gossypii Glover, 1877 & $\begin{array}{l}\text { Punica granatum } \\
\text { Senecio sp. }\end{array}$ & $\begin{array}{l}\text { Hyalopterus pruni } \\
\text { (Geoffroy, 1762) }\end{array}$ & $\begin{array}{l}\text { Prunus } \\
\text { domestica }\end{array}$ \\
\hline $\begin{array}{l}\text { A. hederae Kaltenbach, } \\
1843\end{array}$ & Hedera helix & $\begin{array}{l}\text { Liosomaphis berberidis } \\
\text { (Kaltenbach, 1843) }\end{array}$ & $\begin{array}{l}\text { Berberis } \\
\text { thunbergii } \\
\text { 'Atropurpurea' }\end{array}$ \\
\hline $\begin{array}{l}\text { A. molluginis (Börner, } \\
\text { 1950) }\end{array}$ & Gallium aparine & $\begin{array}{l}\text { Lipaphis erysimi } \\
\text { (Kaltenbach, 1843) }\end{array}$ & Brassica sp. \\
\hline $\begin{array}{l}\text { A. nasturtii Kaltenbach, } \\
1843\end{array}$ & Rumex sp. & $\begin{array}{l}\text { Macrosiphum } \\
\text { euphorbiae (Thomas, } \\
\text { 1878) }\end{array}$ & Urtica sp. \\
\hline $\begin{array}{l}\text { A. nerii Boyer de } \\
\text { Fonscolombe, } 1843\end{array}$ & Nerium oleander & $\begin{array}{l}\text { M. impatientis } \\
\text { Williams, } 1911\end{array}$ & Rosa canina \\
\hline A. pomi de Geer, 1773 & Pyrus sp. & $\begin{array}{l}\text { M. rosae (Linnaeus, } \\
\text { 1758) }\end{array}$ & Rosa sp. \\
\hline $\begin{array}{l}\text { A. ruborum (Börner, } \\
\text { 1932) }\end{array}$ & Rubus sp. & $\begin{array}{l}\text { Myzus persicae } \\
\text { (Sulzer, 1776) }\end{array}$ & Malva sp. \\
\hline A. spiraecola Patch, 1914 & $\begin{array}{l}\text { Trifolium pratense } \\
\text { Cotoneaster } \\
\text { franchetti } \\
\text { Chenopodium sp. } \\
\text { Nerium oleander }\end{array}$ & $\begin{array}{l}\text { Ovatomyzus } \\
\text { chamaedrysi } \\
\text { (Passerini,1879) }\end{array}$ & Mentha sp. \\
\hline $\begin{array}{l}\text { A. umbrella (Börner, } \\
\text { 1950) }\end{array}$ & Malva neglecta & $\begin{array}{l}\text { Panaphis juglandis } \\
\text { (Goeze, 1778) }\end{array}$ & Juglans regia \\
\hline $\begin{array}{l}\text { Aulocorthum solani } \\
\text { (Kaltenbach, 1843) }\end{array}$ & Ulmus sp. & $\begin{array}{l}\text { Patchiella reaumuri } \\
\text { (Kaltenbach, 1843) }\end{array}$ & Tilia sp. \\
\hline $\begin{array}{l}\text { Brachycaudus cardui } \\
\text { (Linnaeus, 1758) }\end{array}$ & $\begin{array}{l}\text { Carthamus sp. } \\
\text { Carduus acanthoides }\end{array}$ & $\begin{array}{l}\text { Rhopalosiphum padi } \\
\text { (Linnaeus,1758) }\end{array}$ & $\begin{array}{l}\text { Hordeum } \\
\text { murinum }\end{array}$ \\
\hline $\begin{array}{l}\text { B. helichrysi (Kaltenbach, } \\
\text { 1843) }\end{array}$ & Cynoglossum sp. & $\begin{array}{l}\text { Schizaphis } \\
\text { rotundiventris } \\
\text { (Signoret, 1860) }\end{array}$ & Palm sp. \\
\hline Brevicoryne brassicae & Brassica sp. & Sitobion avenae & Cynodon \\
\hline
\end{tabular}




\section{(Linnaeus, 1758)}

Chaitophorus populialbae

(Boyer de Fonscolombe,

1841)

Cinara tujafilina del

Guercio, 1909

C. brauni Börner, 1940

C. maghrebica Mimeur,
Populus alba

Platycladus

orientalis

Pinus sp.
(Fabricius, 1775)

S. fragariae (Walker, 1848)

Uroleucon sonchi

(Linnaeus, 1767)

Wahlgreniella nervata

(Gilette, 1908) dactylon

Hordeum sp.

Poa sp.

Bromus sp.

Chondrilla

junce

Sonchus sp.

Pinus sp.

*These new records were listed in the checklist (Görür et al., 2012)

Aphid species and related host plants of Büyükada island are reported here for the first time. 43 species of aphids belonging to 23 genera from 46 different host plants only in Büyükada were recorded. Eulachnus pumilae Inouye, 1939 and Acyrthosiphon kondoi Shinji, 1938 were described as new additions to aphidofauna of Turkey. In Yalova, southern province of Büyükada, 21 aphid taxa placed in 13 genera were identified. Aphis fabae Scopoli 1763 and A. gossypii Glover, 1854 are the most common species, like our study (Kuloğlu \& Özder, 2017). Among aphid population collected host plants Malva neglecta, Nerium oleander and Pinus spp. are the most sampled plant species. Aphis craccivora, Aphis fabae and A. spiracola are the most common and important pests for several plant species. This aphid species affects plants by direct or indirect damage. Therefore, the determining of $t$ these species and their distribution areas are crucial for the control of aphids in Büyükada islands.

The determination of the aphid fauna and host plants of aphids from the study area are clearly indicated how it is important to conduct such detailed study to find out aphid-host plant relations in Turkey. Büyükada (İstanbul) has plant richness and different microclimatic areas and is isolated from the mainland. Number of the determined aphid species and collected host plants and new records are in accordance with the findings in neighboring areas and countries near to the study area (Kuloğlu \& Özder, 2017; Kök \& Kasap, 2019). Görür et al. (2020) pointed out that even listing about 570 aphid species on about 1100 plant species by analyzing 13.000 samples, findings do not adequately figure out real composition of Turkey aphid fauna. Turkey has its own particular features (floristic and faunistic richness) that directly impact aphid diversity and distributions. In the neighboring countries, number of aphid fauna (Greece, 335; Iran, 328; Georgia, 320) is close to Turkey aphid composition but host plant diversity in Turkey is more (Barjadze, Japoshvili, \& Bakhtadze, 2010; Alikhani, Rezwani, Rakhshani \& Madani, 2010; Margaritopoulos, Papapanagiotou, Voudouris, Kati \& Blackman, 2013; Güner, Aslan, Ekim, Vural \& Babac, 2012).

\section{Conclusion}

Due to their reproduction style, invading new areas and resulting in considerable amount of decrease in agricultural production, aphids fascinate researchers. Despite these facts, there are still many places that their aphid fauna has not been observed yet in Turkey. This study is the first detailed scientific effort dealing with the aphid species and collected host plants of Büyükada island of İstanbul region. Furthermore, because of global climate change, tourism, agricultural activities, increasing international trades and transport over the last two decades, introduction of non-native species, including aphids has increased (Kollar \& Barta, 2016). Büyükada is one of the most important tourism center of Istanbul. So non-native host plants and aphid species are easily introduced and distributed in all parts of the island (Kantarc1, 1984). Büyükada has impressive natural and exotic plant species so this introduction can be damage to them. Because Turkey has several natural areas such as Büyükada Island, the findings of the presented study strongly encourage researchers to carry out similar studies. 


\section{Acknowledgement}

These results are a part of Hayal AKYILDIRIM BEĞEN's Master Science Thesis and some part of this study published IVth Turkey Plant Protection Congress. This study is a original research article.

\section{Author Contributions}

Hayal AKYILDIRIM BEĞEN: Investigation, collected data, performed the analysis and wrote the paper.

Gazi GÖRÜR: Investigation, resource, review and wrote the paper.

\section{Conflicts of Interest}

The authors declare no conflict of interest.

\section{References}

Akyürek, B., Zeybekoğlu, Ü. \& Görür, G. (2010). New records of aphid species (Hemiptera: Aphidoidea) for Turkish fauna from Samsun province. Turkish Journal of Zoology, 34, 421424. Retrieved from: http://journals.tubitak.gov.tr/zoology/issues/zoo-10-34-3/zoo-34-3-18-081016.pdf

Akyürek, B., Zeybekoğlu, Ü. \& Görür, G. (2011). Further contributions to the Turkey Aphid (Hemiptera: Aphidoidea) Fauna. Journal of Entomological Research Society, 13 (3), 101-106. Retrieved from: https://www.researchgate.net/publication/289078208_Further_contributions_to_the_Turkey_Aphid_H emiptera Aphidoidea Fauna

Alikhani, M., Rezwani, A., Rakhshani, E. \& Madani, S.M.J. (2010). Survey of aphids (Hem., Aphidoidea) and their host plants in central parts of Iran. Journal of Entomological Research, 2 (2), 7-16. Retrieved from: https://www.researchgate.net/publication/230839002

Barjadze, S., Japoshvili, G. \& Bakhtadze, N. (2010). New records for the Geogian aphid fauna (Hemiptera, Aphididae). Zoology in the Middle East, 50, 140-141. Retrieved from: https://www.researchgate.net/publication/248092122 New records for the Georgian Aphid fauna

Barjadze, S., Özdemir, I. \& Blackman, R. (2014). Two new species of Aphidini Latreille, 1802 (Hemiptera: Aphididae) from Turkey. Zootaxa, 3873(2). Retrieved from: https://www.biotaxa.org/Zootaxa/article/view/zootaxa.3873.2.6

Barjadze, S., Blackman, R. \& Özdemir, I. (2015). A new species of Aphis Linnaeus (Hemiptera, Aphididae) living on Teucrium polium L. (Lamiaceae). Zootaxa, 4057 (2): 248-256. Retrieved from: https://pubmed.ncbi.nlm.nih.gov/26701477/

Blackman, R.L. \& Eastop, V.F. (2020). Aphids of the world's plants. An online identification and information guide. Retrieved from: http://www.aphidsonworldsplants.info . Downloaded on 28 February 2020.

Çanakçıŏlu, H. (1975). The Aphidoidea of Turkey. İstanbul: Istanbul University Faculty of Forestry Publication. Retrieved from: https://www.nadirkitap.com/the-aphidoidea-of-turkeyimzali-hasan-canakcioglu-kitap654139.html 
Eser, S.İ., Görür, G., Tepecik, İ. \& Akyıldırım, H. (2009). Aphid (Hemiptera: Aphidoidea) species of the Urla district of İzmir region. Journal of Applied Biological Sciences, 3 (1), 99-102. Retrieved from: http://www.jabsonline.org/index.php/jabs/article/view/124

Fahringer, J. (1922). Eine Rhynchotenausbeute aus der Türkei, Kleinasien und den benachbarten Gebieten. Konovia 1, 296-307. Retrieved from: https://www.zobodat.at/pdf/KON_1_02960307.pdf

Favret, C. (2020). Aphid species file (Version 5.0/5.0). (Web page: aphid.speciesfile.org) (Date accessed: November 2018). Retrieved from: http://aphid.speciesfile.org/HomePage/Aphid/HomePage.aspx

Görür, G., Zeybekoğlu, U., Akyurek, B., Isık, M. \& Akyıldırım, H. (2009). Trabzon, Rize ve Artvin Illerinin Afit (Homoptera: Aphididae) Faunasinın Belirlenmesi. Tubitak Projesi, Proje numaras1: 107T450.

Görür, G., Akyıldırım Beğen, H., Olcabey, G. \& Akyurek, B. (2012). The aphid fauna of Turkey: An updated checklist. Archives of Biological Science Belgrade, 64 (2), 675-692. Retrieved from: https://www.researchgate.net/publication/267337017

Görür, G., Toper Kaygin, A., Şenol, Ö. \& Akyildirim Beğen, H. (2015). Cinara curvipes (Patch, 1912) (Hemiptera; Aphididae) as new aphid species for Turkish aphidofauna. Artvin Coruh University Journal of Forestry Faculty, 16, 37-39. Retrieved from: https://www.researchgate.net/publication/280244383

Görür, G., Şenol, Ö. \& Akyıldırım Beğen, H. (2019). Adıyaman, Malatya ve Şanlıurfa İllerinden Belirlenen Afit Türlerinin Türkiye Afit Faunasına Katkıları Açısından Değerlendirilmesi. Selçuk Üniversitesi Fen Fakültesi Dergisi, 45(2), 103-115. Retrieved from: https://www.researchgate.net/publication/336901673

Görür, G., Şenol, Ö., Akyıldırım Beğen, H. \& Akyürek, B. (2020). Foresights Derived from Recent Studies Conducted on Turkey Aphid Fauna. Atatürk University Journal of Agricultural Faculty, 51 (1), 63-68. Retrieved from: https://dergipark.org.tr/tr/pub/ataunizfd/issue/52065/597925

Güner, A., Aslan, S., Ekim, T., Vural, M. \& Babaç, M.T. (2012). Türkiye Bitkileri Listesi (Damarlı Bitkiler). Nezahat Gökyiğit Botanik Bahçesi ve Flora Araştırma Derneği Yayını, İstanbul. Retrieved from: https://www.researchgate.net/publication/273136494

Holman, J. (2009). Host Plant Catalog of Aphids, Palearctic region. Springer, Branisovska, Retrieved from: https://www.springer.com/gp/book/9781402082863

Kantarc1, M.D. (1984). İstanbul Adalarının Yetişme Ortamı Özellikleri, Peyzajı ve Ağaçlandırılması Konusunda Bir İnceleme. Istanbul Universitesi Orman Fakültesi Dergisi Seri B, 34, 49-69. Retrieved from: https://forestist.org/en/istanbul-adalarinin-yetisme-ortamiozellikleri-peyzaji-ve-agaclandirilmasi -konusunda-bir-inceleme-132624

Kaya, B. \& Aladağ, C. (2009). Maki ve Garig Topluluklarının Türkiye'deki Yayılış Alanları ve Ekolojik Özelliklerinin İncelenmesi. Selçuk Üniversitesi Sosyal Bilimler Enstitüsü Dergisi, 22, 67-80. Retrieved from: https://www.researchgate.net/publication/41484787 
Kollar, J. \& Barta, M. (2016). The first record of tulip tree aphid, Illinoia liriodendri (Hemiptera: Aphididae), from Slovakia. Plant Protection Science, 52 (2), 142-146. Retrieved from: https://www.researchgate.net/publication/299418737

Kök, Ş., Kasap, İ. \& Özdemir I. 2016. Aphid (Hemiptera: Aphididae) species determined in Çanakkale Province with a new record for the aphid fauna of Turkey. Turkish Journal of Entomology, 40 (4): 397-412. Retrieved from: https://dergipark.org.tr/tr/download/article-file/269965

Kök, Ş. \& Kasap, İ. 2019. Aphid (Hemiptera: Aphididae) species of the South Marmara Region of Turkey including the first record of Dysaphis radicola meridialis Shaposhnikov, 1964 for the aphid fauna of Turkey. Turkish Journal of Entomology, 43 (1): 63-78. Retrieved from: https://dergipark.org.tr/tr/download/article-file/668506

Kuloğlu, I. \& Ozder, N. (2017). Aphids (Hemiptera: Aphididae) on Ornamental Plants from Yalova Province, Turkey. COMU Ziraat Fakultesi Dergisi, 5 (2): 69-72. Retrieved from: https://dergipark.org.tr/tr/pub/comuagri/issue/33321/333141

Margaritopoulos, J.T., Papapanagiotou, A.P., Voudouris, C.H., Kati, A. \& Blackman, R.L. (2013). Two aphid species newly introduced in Greece. Entomologia Hellenica, 22, 23-28. Retrieved from: https://www.researchgate.net/publication/320938760

Martin, J.H. (1983). The identification of common aphid pests of tropical agriculture. Tropical Pest Management, 29, 395-411. Retrieved from: https://www.tandfonline.com/doi/abs/10.1080/09670878309370834

MGM (2020). Büyükada iklim verileri. https://mgm.gov.tr/tahmin/il-veilceler.aspx ?il=ISTANBUL\&ilce=BUYUKADA\#/ Downloaded on 11.04.2020.

Remaundière, G., Toros, S. \& Özdemir, I. (2006). New contribution to the aphid fauna of Turkey (Hemiptera:Aphidoidea). Revue Française d'Entomologie, 28, 75-96. Retrieved from: https://www.semanticscholar.org/paper/

Şenol, O., Akyıldırım Beğen, H., Gorur, G. \& Demirtaş, E. (2015a). New additions and invasive aphids for Turkey's Aphidofauna (Hemiptera: Aphidoidea). Turkish Journal of Zoology, 39, 39-45. Retrieved from: https://www.researchgate.net/publication/267336891

Şenol, Ö., Akyıldırım, H., Görür, G., Demirtaş, E. (2015b). New entry for the Turkey aphidofauna [Hemiptera: Aphidoidea]. Acta Zoologica Bulgarica, 66 (1), 133-136. Retrieved from: https://www.researchgate.net/publication/267336790

Toper Kaygin, A., Gorur, G. \& Cota, F. (2008). Contribution to the Aphid (Homoptera: Aphididae) species damaging on woody plants in Bartın, Turkiye. International Journal of Engineering Science, 2 (1), 83-86. Retrieved from: http://ijnes.org/index.php/ijnes/article/view/382 